\title{
Pipeline Reduction of Binary Light Curves from Large-Scale Surveys
}

\author{
Andrej Prša ${ }^{1,2}$ and Tomaž Zwitter ${ }^{2}$ \\ ${ }^{1}$ Villanova University, Dept. of Astronomy, 800 Lancaster Ave, Villanova, PA 19085, USA \\ ${ }^{2}$ University of Ljubljana, Dept. of Physics, Jadranska 19, SI-1000 Ljubljana, EU \\ emails: andrej.prsa@fmf.uni-lj.si, tomaz.zwitter@fmf.uni-lj.si
}

\begin{abstract}
One of the most important changes in observational astronomy of the $21^{\text {st }}$ Century is a rapid shift from classical object-by-object observations to extensive automatic surveys. As CCD detectors are getting better and their prices are getting lower, more and more small and medium-size observatories are refocusing their attention to detection of stellar variability through systematic sky-scanning missions. This trend is aditionally powered by the success of pioneering surveys such as ASAS, DENIS, OGLE, TASS, their space counterpart Hipparcos and others. Such surveys produce massive amounts of data and it is not at all clear how these data are to be reduced and analysed. This is especially striking in the eclipsing binary (EB) field, where most frequently used tools are optimized for object-by-object analysis. A clear need for thorough, reliable and fully automated approaches to modeling and analysis of EB data is thus obvious. This task is very difficult because of limited data quality, non-uniform phase coverage and solution degeneracy. This paper reviews recent advancements in putting together semi-automatic and fully automatic pipelines for EB data processing. Automatic procedures have already been used to process Hipparcos data, LMC/SMC observations, OGLE and ASAS catalogs etc. We discuss the advantages and shortcomings of these procedures.
\end{abstract}

Keywords. methods: data analysis, numerical; catalogues, surveys; binaries: close, eclipsing, fundamental parameters; techniques: photometric, spectroscopic

\section{Introduction}

Doing astronomy today is simply unimaginable without computers. To facilitate observing preparations, we use databases; to observe, we use control software; to reduce the acquired data, we use reduction programs. Just how far the computer autonomy of the data acquisition process goes is best described by the increasing trend of refurbrishing small and medium-size telescopes into fully automatic, robotic instruments $\dagger$. Surveys such as OGLE (Udalski et al. 1997), EROS (Palanque-Delabrouille et al. 1998), ASAS (Pojmanski 2002), space mission Hipparcos' epoch photometry (Perryman \& ESA 1997), and others, have changed observational astronomy: streams of data produced by automatic telescopes around the world and in space are overwhelming for currently existing tools and astronomers cannot cope anymore.

Take eclipsing binaries, for example. So far there have been about 500 published papers with physical and geometrical parameters determined to better than $3 \%$ accuracy. For a skilled eclipsing binary guru it takes 1-2 weeks to reduce and analyse a single eclipsing binary by hand. To date, there are about 10000 photometric/RV data-sets that in principle allow modeling to a 3\% accuracy. By 2020, the upcoming missions such as Pan-Starrs (Kaiser et al. 2002) and Gaia (Perryman et al. 2001) will have pushed this

$\dagger$ A comprehensive list of more than a hundred such facilities may be found, e.g., at

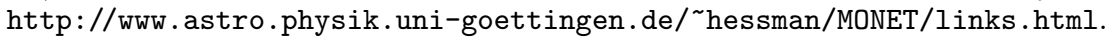



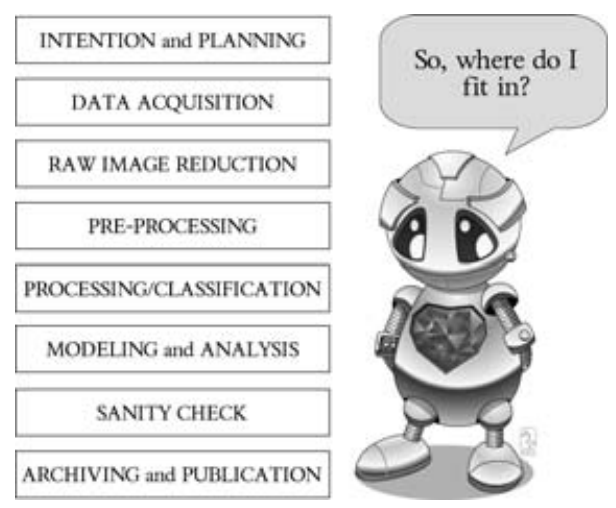

Figure 1. Schematic view of a typical EB reduction and analysis pipeline.

number to $\sim 10000000$. Even if all observational facilities collapsed at that point so that no further data got collected, it would take 12500 astronomers to analyse these data in the next 100 years! Since there are currently about 13000 members of the IAU, the only way to achieve this in the next 100 years by traditional methods is to have every astronomer in the world doing eclipsing binaries. And of course, do not forget to shut down all robotic telescopes out there!

With the change in observational astronomy, traditional analysis methods and tools need to change too. This paper overviews most important aspects of automatic procedures, tiers that form a pipeline reduction of eclipsing binary light curves. Next Section deals with basic principles of the reduction and analysis pipeline; Section 3 reviews most important applications of automatic pipelines on large-scale survey data. Section 4 stresses the everlasting importance of dedicated observations. Finally, Section 5 concludes and gives some prospects for the future.

\section{Tiers of the reduction and analysis pipeline}

A full-fledged pipeline for reduction and analysis of photometric data of eclipsing binary stars would ideally consist of 8 distinct tiers depicted in Figure 1.

\subsection{Intention and planning}

For as long as we discuss stellar objects in general, and eclipsing binaries in particular, there are two apparently frightening facts that need to be considered: $\mathbf{1}$ ) a target star has already been observed and 2) a target star has already been observed many times. There are literally hundreds of photometric survey missions that have been swiping the sky across and over in a very wide magnitude range, and chances are indeed slim that a given star has not been observed yet.

According to Hipparcos results, there are about $0.8 \%$ of eclipsing binaries in the overall stellar population (917 out of 118218 stars, Perryman \& ESA 1997). Projecting these statistics to other large surveys gives an estimate of how many eclipsing binaries are expected to be present in survey databases: $\sim 136000$ in ASAS (11076 detected by Paczyński et al. 2006), 56000 in the OGLE LMC field (2 580 detected by Wyrzykowski et al. 2003), 16000 in OGLE SMC field (1350 detected by Wyrzykowski et al. 2004), $\sim 80000$ in TASS (Droege et al. 2006) etc. Gaia will make a revolution in these numbers since the aimed census of the overall stellar population is $\sim 1$ billion up to $V=20$ (Perryman et al. 2001). Admittedly, magnitude levels and variability detection threshold change from survey to survey, but a shortage of eclipsing binaries in the databases is 


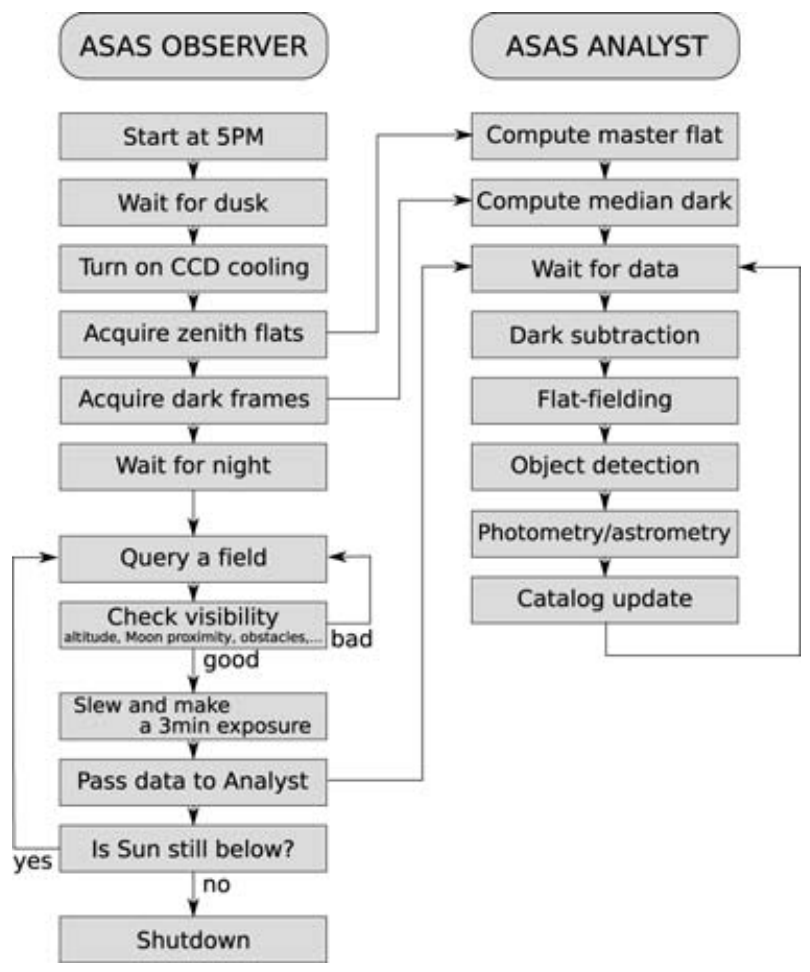

Figure 2. Automatic pipeline of the ASAS project. The pipeline consists of two separate (yet connected) engines: Observer and Analyst. The Observer takes care of the data acquisition, and the Analyst takes care of data reduction and analysis. The only human intervention needed is closing the observatory in case of bad weather and changing the DAT-2 storage tapes. The schematic view was adapted from the description of the ASAS project (Pojmanski 1997).

more than obvious. In other words, there are many eclipsing binaries out there that are either undetected, unconfirmed or misclassified. Stressing a well-known fact that eclipsing binaries are unique in their potential to yield accurate masses, radii, temperatures and distances, and realizing that many of them are reachable by small-size ground instruments, eclipsing binaries should definitely hold one of the top positions on observational candidates list.

\subsection{Data acquisition}

Most automated of all pipeline tiers, data acquisition has become a truly reliable runof-the-mill. An example of a fully automatic data acquisition and analysis pipeline is that of the All-Sky Automated Survey (ASAS, Pojmanski 1997), depicted on Figure 2. The level of sophistication is already such that it assures accurate and reliable data both from ground-based and space surveys - and in plenty. A more serious problem for space surveys seems to be telemetry: how do we get the data down to Earth? For instance, Hipparcos' downlink rate was only $24 \mathrm{kbit} / \mathrm{s}$, Gaia's will be $5 \mathrm{Mbit} / \mathrm{s}$ (Lammers 2005) significantly less than the bandwidth we are used to from everyday life. To avoid using lossy compression algorithms, surveys must use optimized telemetry for the given field and/or data pre-processing (e.g. binning, filtering, selective downloads). Reliable and lossless I/O pipelines and finding ways to store all the acquired data are definitely two of the greatest challenges for data acquisition of the future. 


\subsection{Raw image reduction}

Acquired data must be reduced: two-dimensional images must be converted to the observed quantity (magnitudes, fluxes, ....). To fully appreciate the need for accurate image reduction, one must consider a multitude of physical and instrumental effects that influence the observed data. Some of them - e.g., telescope optics, CCD quantum efficiency and non-linearity, filter response - may be adequately treated during the reduction process. Others - sky variability, instrumental temperature dependence, cosmic rays, interstellar and atmospheric extinction - usually demand more involvement because of their dependence on time and wavelength, or because of unknown physical conditions. Raw image reduction consists of taking the acquired image, extracting the data and removing all instrumental artifacts contained in that data. This procedure, along with the developed tools (e.g., IRAF, Tody 1986), relies somewhat on human intervention, but in principle it could be automated to meet the accuracy of today's surveys. One of the steps in the ASAS pipeline, for example, is a fully automated reduction (c.f., Figure 2): subtracting dark current and flat-fielding (Pojmanski 1997).

\subsection{Pre-processing}

Once the images have been reduced, the data are ideally free from instrumental systematics, but imprints of other effects (most notably atmospheric extinction and variable seeing) in phased data are still present. These effects may be significant and, as such, they should be removed from the data. To this procedure we refer to as pre-processing.

There are two approaches to pre-processing: parametric modeling and detrending. The former uses modeling functions and seeks optimal parameters to reproduce the effect at hand; since it relies on physical insights, its application is more-or-less transparent. Detrending, on the other hand, is based on statistical properties of the observed time series and uses mathematical tools to achieve the same goal. Treating atmospheric extinction with parametric models is given e.g., by Prša \& Zwitter (2005b), while detrending is presented e.g. by Tamuz et al. (2005). Since the application of the latter is not limited to just a given physical effect, it is well worth stressing its major strengths.

Strictly speaking, a trend in a time series is a slow, gradual change in observables that obscures parameter relationships under investigation. Detrending is a statistical operation of removing stochastical dependence in consecutive observations, thus making the pre-processed data distributed according to the normal (Gaussian) probability distribution function. Tamuz et al. (2005) proposed a generalized Principal component analysis (PCA) method that accounts for variable observation uncertainties. The method is able to remove systematics from the data without any prior knowledge of the effect. Figure 3 shows an example of how the algorithm is able to process noisy planetary transit data (top row) by consecutively detrending four distinct systematic effects, yielding the detrended data (bottom row). Strengths of the method are its universality and little importance of the starting values of trend parameters, and the reduction to ordinary PCA in case of constant observation uncertainties. Its deficiencies are non-orthogonal eigenvectors (and thus deteriorated statistical properties in cases of a highly variable $\mathrm{S} / \mathrm{N}$ ratio), a danger of filtering out intrinsic long-term variability and no relation to physical background of the trend. That said, generalized PCA method has proven to be one of the most successful methods for detrending that has been applied so far.

\subsection{Processing/classification}

By the time the observed data is ready for scientific munching, most of the non-intrinsic artifacts should have been removed. By processing we refer to seeking broad scientific properties of the observed object: analysis of variance, period determination, phased curve 
Transit 1
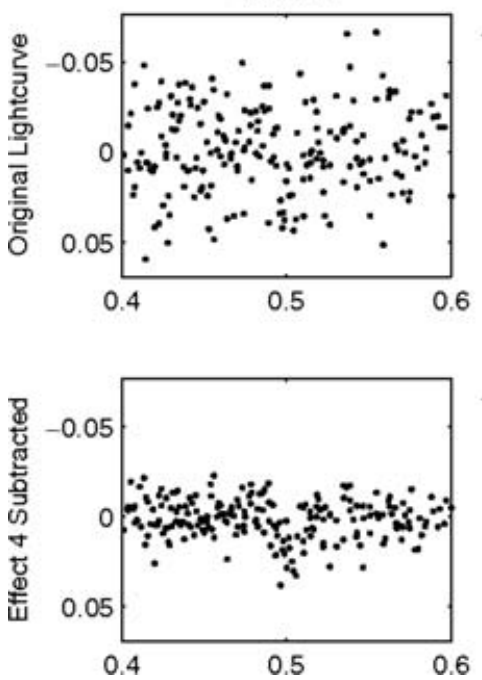

Transit 2

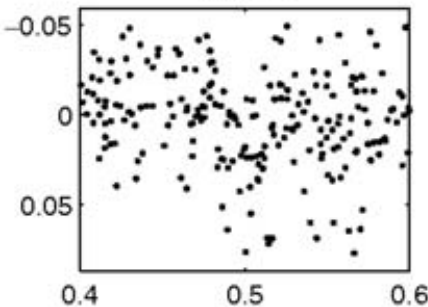

Transit 3
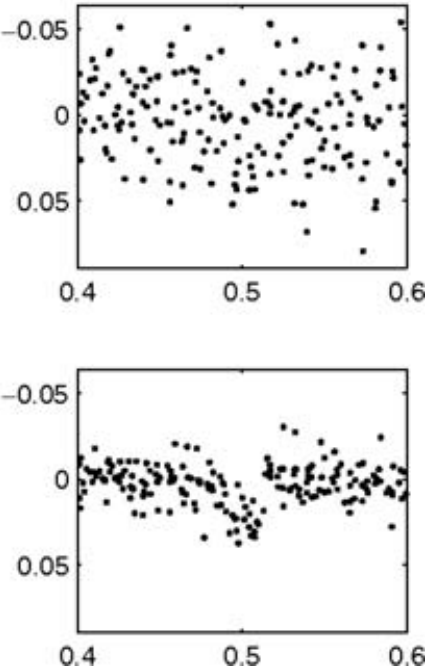

Figure 3. Detrending results for 3 planetary transit examples. The plotted diagrams depict relative magnitude vs. phase. The top row shows the original data, and the bottom row shows the detrended data, after 4 consecutive detrending iterations. Adapted from Tamuz et al. (2005).

folding etc. Closely related is classification: based either on the processing results or on statistical pattern analysis, the observed objects are classified into their respective groups. While manual approaches usually rely on the former principle (we recognize the shape of the light curve and evaluate it critically in a broader physical context - do parameters make sense, is the period plausible for a given type of object, ...), automatic approaches will prefer the latter principle, e.g., through the use of Fourier fitting, inversions, neural networks etc. Either way, processing and classification aim to discriminate gems from ordinary rocks in terms of our primary interest.

\subsection{Modeling and analysis}

Computationally most demanding task, at least with respect to eclipsing binaries, is their modeling and analysis. Seeking and interpreting a set of physical and geometrical parameters involves solving the inverse problem. There are many dedicated codes that enable accurate modeling - WD (Wilson \& Devinney 1971), WINK (Wood 1971), NDE (Nelson \& Davis 1972), EBOP (Etzel 1981), FOTEL (Hadrava 1990) and many others. We discuss their usage and application to survey data in detail in the following section.

\subsection{Sanity check}

A famous statement by R.E. Wilson, "There is more to modeling eclipsing binaries than parameter fitting," pretty much encapsulates the idea of sanity check. Solving the inverse problem does not only mean finding physical and geometrical parameters that best reproduce the data, it also means seeking parameter inter-dependencies, understanding hyperspace non-linearity and, above all, being aware of the limitations of the data-set at hand and the used modeling engine. Since eclipsing binaries are used for "calibrating the calibrations", mis- and over-interpreting the data may have tragic consequences on solution reliability. Getting a solution from a model is only a fraction of the work; the majority is assessing its uniqueness and physical feasibility of that solution. 


\subsection{Archiving and publication}

More important than the publication of papers themselves is the question on publishing data. What to do with the immense data flow that is expected from large-scale surveys? How to set standards and specifications for publishing and storing data? How to coordinate efforts and how to distribute the results? Finally, what is our next step in terms of model enhancements? Let us face it - missions such as CoRoT (Baglin et al. 2002) and Kepler (Koch et al. 2004) will deliver milli-magnitude accuracies in just a few years - do we honestly believe that our models can support such accuracies? All of these are still open questions that demand our immediate attention.

\section{First bites on large databases}

One of the first attempts to survey eclipsing binaries in the LMC goes back to PayneGaposchkin (1971), who visually examined about 2000 photographic plates, and classified and listed the main characteristics of 78 eclipsing binaries. At that time computers only started infiltrating modern astronomy and automatic handling was not possible. Yet at the same time, the first EB modeling codes were emerging, most notably those of Horák (1966, 1970), Wilson \& Devinney (1971), Wood (1971), Nelson \& Davis (1972), Mochnacki \& Doughty (1972) and somewhat later Hill (1979), Etzel (1981), Hadrava (1990) and Linnell \& Hubeny (1994), that would eventually form the base of automatic pipelines.

In the early nineties, surveys began to yield first databases that were used for EB detection and analysis. Grison et al. (1995) assembled a list of $79 \mathrm{EBs}$ in the bar of the LMC from the EROS survey data. Of those, only one system was previously identified as an EB, so this work effectively doubled the number of known EBs in the LMC. In the year that followed, Friedemann et al. (1996) used IRAS data (Neugebauer et al. 1984) to look for coincidences in the positions of EBs taken from the $4^{\text {th }}$ edition of the GCVS (Kholopov et al. 1992) and about 250000 IRAS sources. They found 233 candidates, of those $63 \%$ Algol-type binaries where accretion disks could be responsible for the IR imprint.

Attacks on LMC continued by Alcock et al. (1997), who used the MACHO database (Cook et al. 1995) to analyse 611 bright EBs. The selection was based on visual identification by examining phase plots. They pointed out two physical quantities that, besides inclination, account for most variance in light curves: the sum of relative radii and the surface brightness ratio. For preliminary analysis the authors used the Nelson \& Davis (1972) code and, following the GCVS designation types, they proposed a new decimal classification scheme depicted in Figure 4.

The next survey to provide results for 933 EBs was OGLE (Szymanski et al. 1996). Series of systematic analyses were conducted by Rucinski (1997b,a, 1998) and later Maceroni \& Rucinski (1999); Rucinski \& Maceroni (2001) that stressed the success and importance of the Fourier decomposition technique (FDT) for classification of variable stars. The technique itself - fitting a $4^{\text {th }}$ order Fourier series to phased data curves and mapping different types of variables in Fourier coefficient space (c.f., Figure 5, left) - was first proposed for EBs already by Rucinski (1973) and has been used ever since, most notably for classifying ASAS data (Pojmanski 2002; Paczyński et al. 2006).

Somewhat ironically, the first one to implement a fully automatic analysis pipeline for obtaining physical parameters of EBs was the most vocal advocate against any automated approaches: R.E. Wilson. In their two papers, Wyithe \& Wilson (2001, 2002) carried out an automatic search from $1459 \mathrm{EBs}$ in the SMC detected by OGLE to find ideal distance 

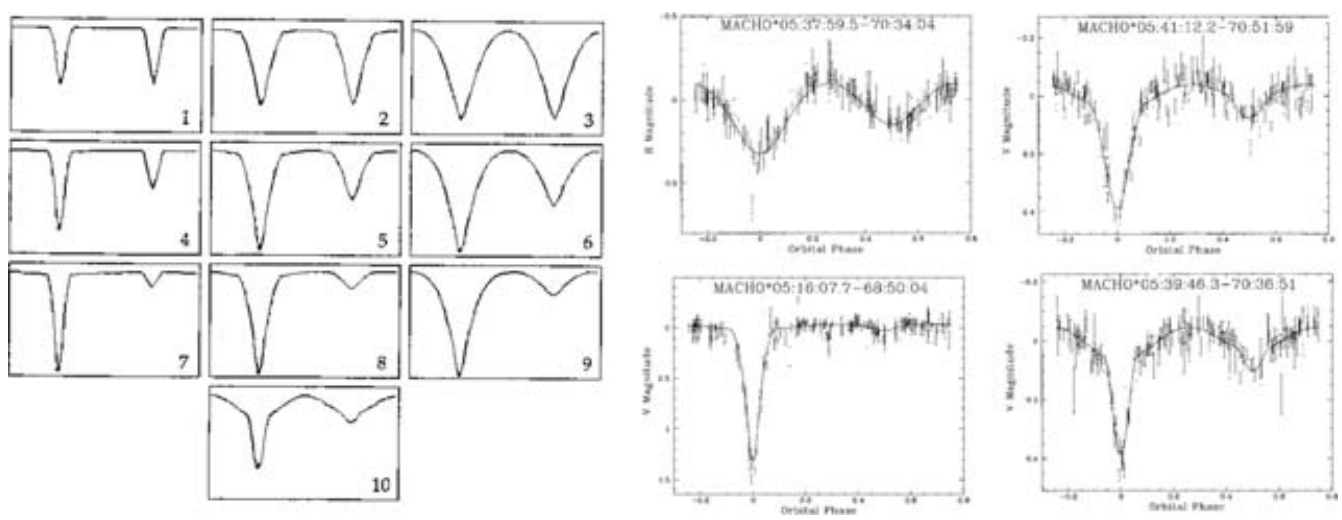

Figure 4. A decimal classification scheme proposed by Alcock et al.. The scheme relies on two physical parameters: the sum of relative radii and the surface brightness ratio. Four plots on the right are classified data from the MACHO survey. Adopted from Alcock et al. (1997).
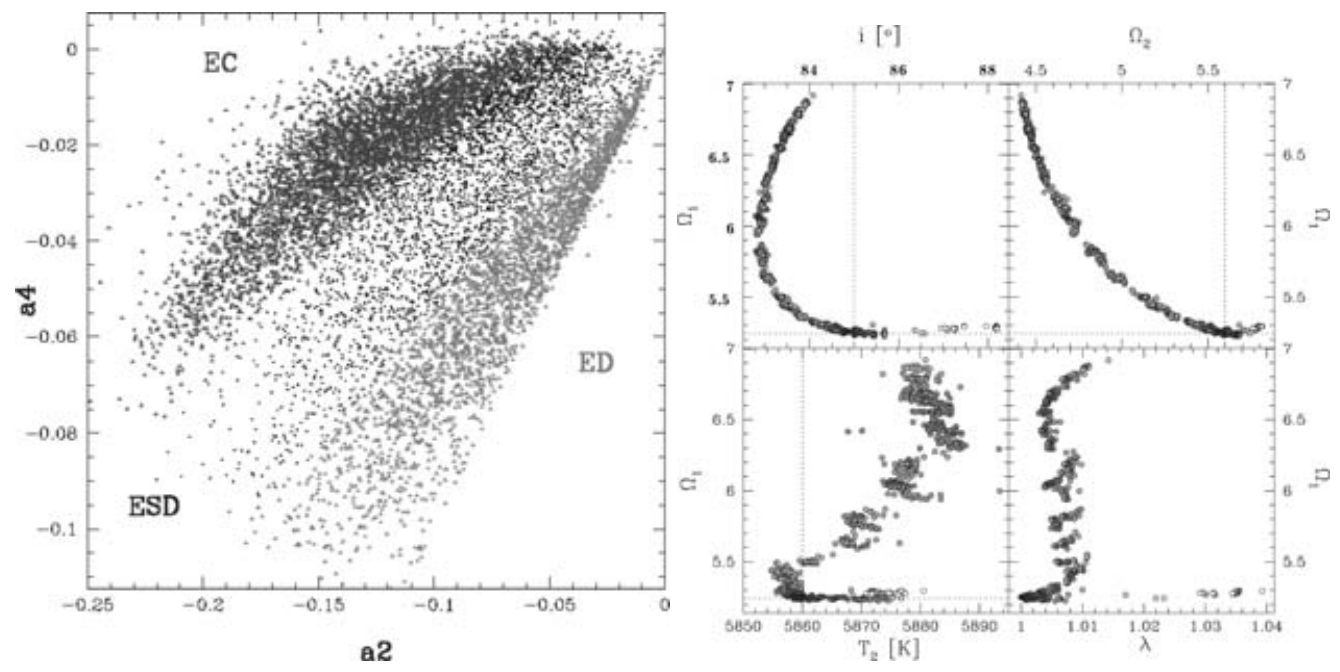

Figure 5. Left: three types of eclipsing binaries (detached, semi-detached, and contact) mapped in the $a_{2}-a_{4}$ Fourier composition space; adopted from Paczyński et al. (2006). Right: heuristic scanning with Powell's direction set method. Converged results are shown for different parameter cross-sections; cross-hairs denote the right solution, and the symbol's shade of gray corresponds to the reached $\chi^{2}$ value: the darker the tone, the lower the $\chi^{2}$. Taken from Prša \& Zwitter (2006).

estimators. WD was run in an automatic mode for the first time, although on a stripped level of complexity: the model assumed canonical values for physical parameters poorly defined by a single-passband photometric data: mass ratio $q=1$, argument of periastron $\omega=0$ or $\pi$, the temperature of the secondary $T_{2}=15000 \mathrm{~K}$, no spots, simple reflection, synchronous rotation etc. Yet for the first time, an automatic, decision-making pipeline was tested against synthetic data and then applied to observations. Despite several deficiencies (systematics introduced through assertions, DC-based method without heuristical search for solution uniqueness, no account of reddening) the authors succeeded to come up with two groups of candidates for ideal distance indicators: widely detached EBs and EBs with total eclipses. A manual follow-up analysis of 19 bright, large-amplitude 


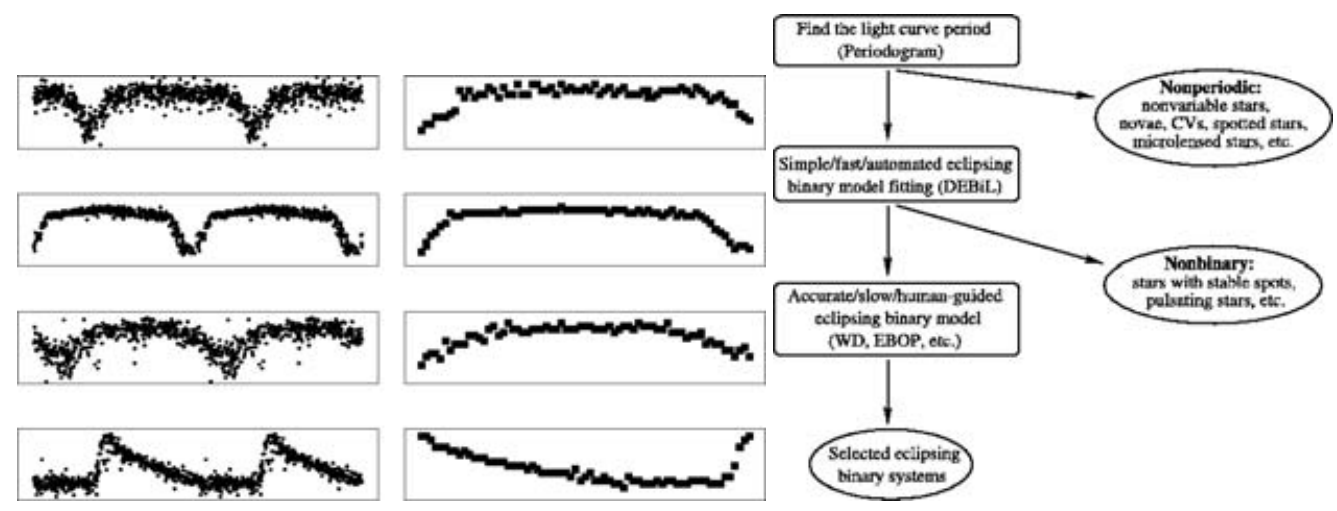

Figure 6. Left: an example of conversion of phased light curves to $70 \times 15$ pixel images, which are fed to the neural network image recognition algorithm. Taken from Wyrzykowski et al. (2003). Right: a tier-based pipeline proposed by Devor (2005): observed light curves are passed sequentially through filters and only the ones that fulfil all criteria make it to the next tier.

candidates in their list was done e.g., by Graczyk (2003), deriving the distance modulus to the SMC to be $\sim 18.9 \pm 0.1$.

Meanwhile, a number of reliable solutions of individual EB solutions was steadily growing by a dedicated series of manual analyses, e.g., by Andersen et al., Munari et al. and others. Instead of immediately going for survey data, our group decided to test fully automatic pipelines on these high-quality data. In our early work (Prša 2003) we obtained encouraging results for 5 morphologically different EBs, stressing importance of data diversity - photometric data without RVs does not suffice for accurate modeling results. Trying to follow up on our devised scheme, we soon identified main deficiencies of the DC algorithm: since it is based on numerical derivatives, it may frequently diverge, and it gets stuck in local minima. To overcome this, we proposed two types of derivative-less methods: Nelder \& Mead's downhill Simplex method (Prša \& Zwitter 2005c) and Powell's Direction set method (Prša \& Zwitter 2006). To understand and explore parameter degeneracy, heuristic scanning and parameter kicking were introduced (Prša \& Zwitter 2005a, c.f., Figure 5, right) - the problem does not lie in the DC, but in the inverse problem itself: its non-linearity, parameter degeneracy and data quality limitations. With this in mind we created a new modeling environment called PHOEBE $\nmid$ (PHysics Of Eclipsing BinariEs; Prša \& Zwitter 2005a) that features a flexible scripting language. This language is developed specifically with modeling and analysis of large surveys in mind.

Continuing with the OGLE data harvest, Wyrzykowski et al. (2003, 2004) identified $2580 \mathrm{EBs}$ in the LMC and $1351 \mathrm{EBs}$ in the SMC. The novelty of their classification approach is using Artificial neural networks (ANN) as an image recognition algorithm, based on phased data curves that have been converted to low-resolution images as depicted on Figure 6. Their classification pipeline was backed up by visual examinations of results. Although there were no physical analyses in their pipeline, observational properties of the sample, as well as 36 distance estimator candidates for the LMC, have been derived.

In 2005, Devor implemented a tier-based elimination pipeline: observed light curves are sequentially passed through filters in the order of increasing computational time cost. Each tier filters out light curves that do not conform to the given criteria. Once a clean sample of light curves is available, it is submitted to a central part of the pipeline, a

$\dagger$ More information on PHOEBE may be found at http://phoebe.fiz.uni-lj.si. 

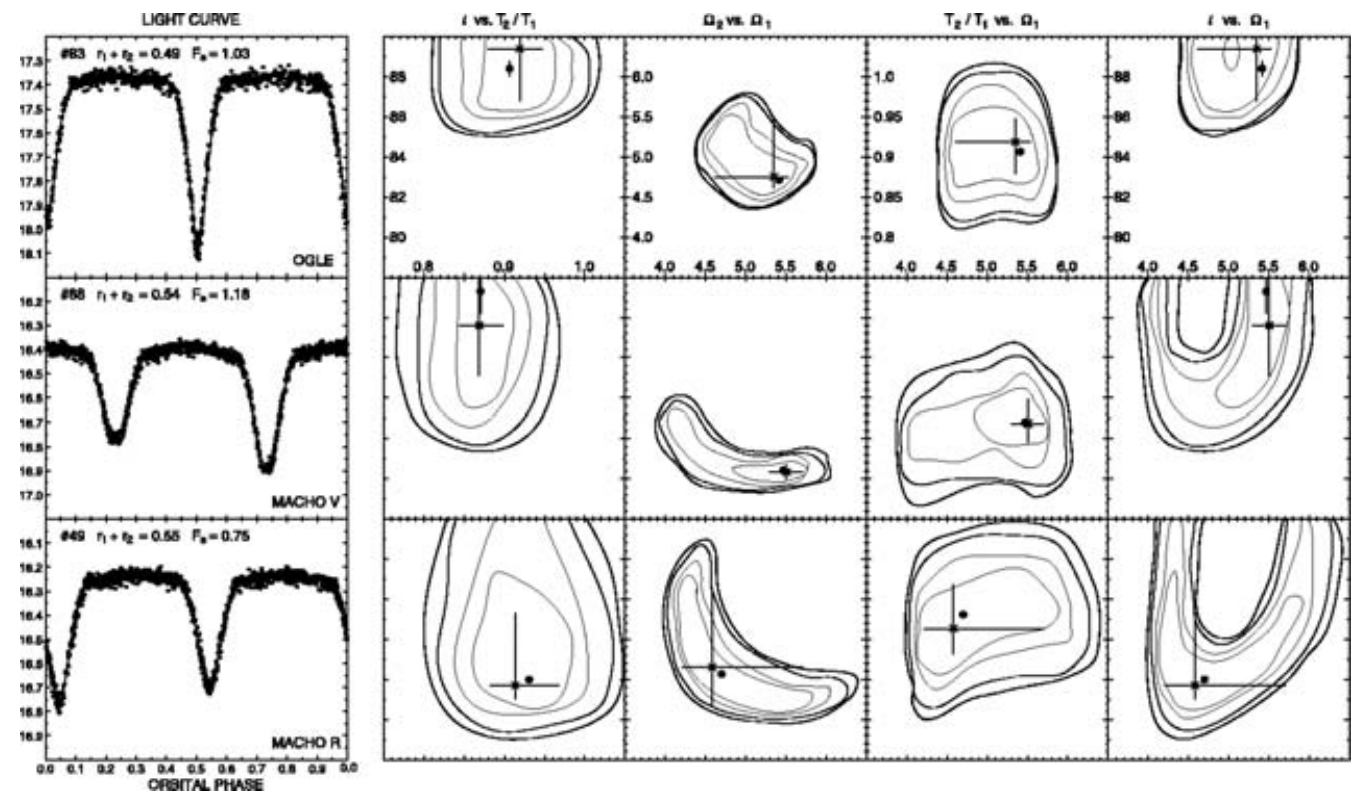

Figure 7. Examples of Monte-Carlo simulations for three EB light curves. Most importantly, the authors stress the importance of examining phase space cross-sections depicted on the right. Please refer to the original paper for further details. Taken from Michalska \& Pigulski (2005).

dedicated program DEBiL (Detached eclipsing binary light curve solver; c.f., Figure 6, right), fitting a simplified EB model (spherical, limb-darkened stars on a classical Keplerian orbit) to observations. The pipeline yielded 10861 eclipsing binaries out of 218699 bulge field variables from OGLE II data (Udalski et al. 1997). Its main advantage is speed: $\sim 1$ minute per light curve on a $333 \mathrm{MHz}$ Sun UltraSparc 5 workstation. Its main deficiencies are lack of the 3rd proposed tier (accurate analysis) and an oversimplified model that may produce false positives among other variables.

One of the best papers, in our opinion, that dealt with eclipsing binaries from OGLE II data, was the one by Michalska \& Pigulski (2005). Its thorough analysis and deep insight into caveats of the EB field make it exemplary for all similar undertakings in the future. The authors limited their analysis to bright $(V<17.5, V-I<0.5)$, high S/N, EA type binaries that exhibit small proximity effects. After proving by example that the original differential image analysis (DIA) calibration is flawed due to uncertainty of reference flux in the flux-to-magnitude calibration, they proposed a novel method of calibrating DIA data and demonstrated its significantly better results. Once the OGLE II data has been re-calibrated, the authors added MACHO, OGLE I and EROS data (when available). The data have been submitted to a WD-based pipeline: the first step was to find initial parameter estimates by the Monte-Carlo method (c.f., Figure 7), and the second step was to converge to the final solution by DC. A result is a list of 98 proposed candidates for distance estimates to the LMC, along with accurately determined parameters in relative units. Out of the sample, 58 stars are found to have eccentric orbits, and 14 systems are exhibiting apsidal motion.

Out of the crowd emerges yet another program to tackle the problem: EBAI (Eclipsing Binaries with Artificial Intelligence; Devinney et al. 2005). This project does not only classify the data, it does more: blindingly fast, it determines coarse parameters of eclipsing binaries in a large data set. Study is underway for these parameters to be fed to a WD-based solver within PHOEBE. This solver maps the hyperspace around the solution, verifying its uniqueness and heuristically determining error estimates. 
Another recent work that we wish to draw specific attention to has been done by Tamuz et al. (2006). The authors devised a new algorithm called EBAS (Eclipsing Binary Automatic Solver), aimed specifically to large datasets and thus based on the faster, yet less accurate EBOP code (Etzel 1981). Similarly to the discussed predecessors, EBAS also uses the sum of relative radii as a principal parameter. Yet there are two important novelties of their approach: instead of inclination the authors introduced the impact parameter - the projected distance between the centers of the two stars during the primary eclipse, measured in terms of the sum of radii - and they introduced a new "alarm" statistics, the goal of which is to automatically discriminate best-fit $\chi^{2}$ values from still apparently acceptable values, but corresponding to distinctively wrong solutions. A follow-up application of EBAS on 938 OGLE LMC binaries with B-type main-sequence primary stars (Mazeh et al. 2006a) yielded the distributions of the fractional radii of the two components and their sum, the brightness ratios and the periods of the short-period binaries. Intriguingly, they observed that the distribution in $\log P$ is flat on the 2-10 days interval and that the detected frequency of their target stars is significantly smaller than the frequency deduced by dedicated RV surveys. The details on these findings are also given by Mazeh et al. (2006b).

Our attempt to preserve paper readability, and struggling against page limits at the same time, regrettably prohibits us to summarize all the work done so far. That is why we wish to at least acknowledge other important developments of this field - and to apologize for any unintentional omissions in this brief review. Reader interested in pipeline reduction of binary light curves from large-scale surveys will surely benefit from the work of Lastennet \& Valls-Gabaud (2002), Brett et al. (2004), Ribas et al. (2004), Wilson (2004), Hilditch et al. (2004, 2005), Eyer \& Blake (2005), Groenewegen (2005), Naficy et al. (2005), Sarro et al. (2006) and many others.

\section{Traditional observations are not obsolete}

After so much stress on surveys, missions and sophistication in fully automatic approaches it is tempting to conclude that traditional object-by-object observations have become obsolete. This is one of most dangerous misconceptions, apparently powered even by our own statement in the introduction that most (if not all) of the candidates have already been observed a number of times. Although these hot topics are appealing because of shear numbers of observed objects, there are several deficiencies in the context of eclipsing binaries that we should be aware of:

- Surveys and missions have a limited life-time that is generally not governed by the eclipsing binary harvest. Rather, limitations arise on account of funding, technology and reaching primary scientific objectives. A direct consequence is the selection effect in observed EBs: only the ones with suitable periods will have been detected.

- The main driving idea of surveys is to acquire as much data as quickly as possible. Due to adopted sky scanning laws, the sky coverage is typically non-uniform and the observations are thus clustered in time. Although this might not seem too important for close binaries, it is critical in case of well detached binaries where there is practically no surface deformation and where eclipses occur only on a narrow phase interval. Having a point or two within the eclipse is hardly any different than having no point at all.

- In order to reach survey completeness in terms of object counts during the mission life-time, the number of data points per object is usually poor. This means that the phase coverage for eclipsing binaries is often not sufficient for recognition and classification purposes, because of the strong sensitivity of period detection algorithms to phase completeness. 


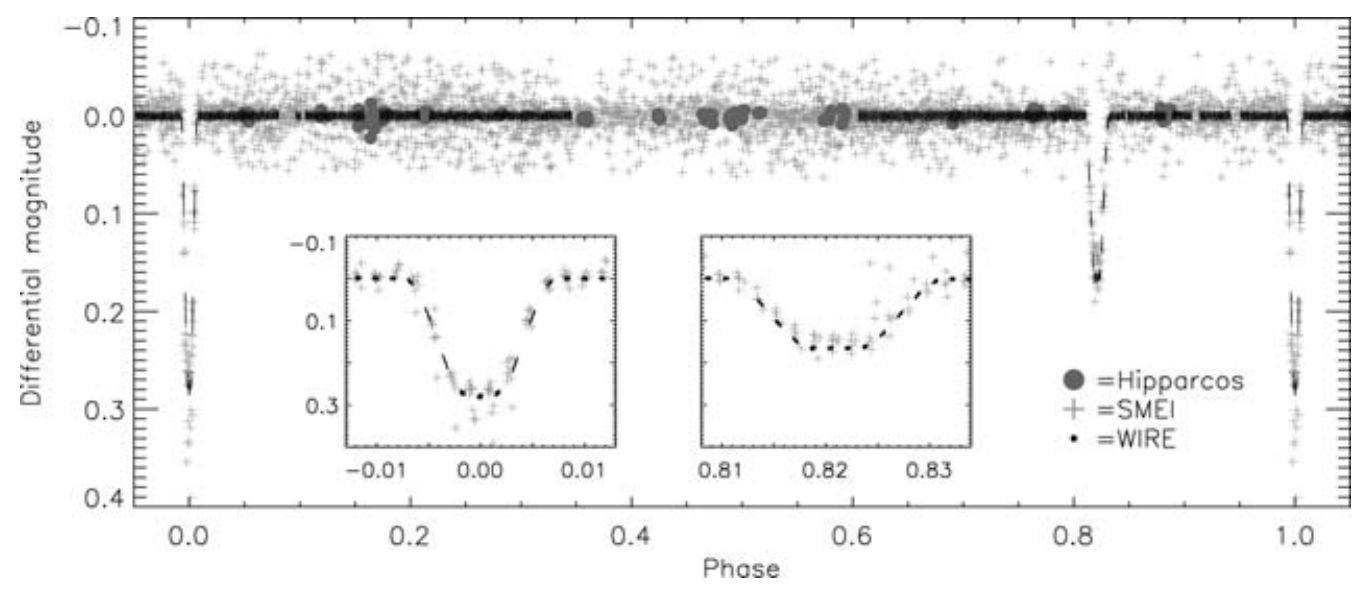

Figure 8. Phased light curve of $\psi$ Cen. Data points from WIRE (black dots) may be compared to those from SMEI (gray plus symbols) and from Hipparcos (grey circles). Taken from Bruntt et al. (2006).

- Unprecedented in numbers does not mean unprecedented in accuracy. At least so far, survey data has been more challenging to reduce and analyse than a typical dedicated observation because of the significant data scatter. However, WIRE (the Wide-field InfraRed Explorer, Hacking et al. 1999), despite its failure to achieve primary scientific goals, had already given us a snapshot of the milli-magnitude photometric precision of the future (c.f., Figure 8).

- Most importantly: surveys usually lack data diversity. In order to get absolute temperatures of both stars and interstellar extinction, multi-passband photometry is needed; to get reliable estimates of absolute sizes of an eclipsing binary system, radial velocities are needed. To break inter-parameter correlations and solution degeneracy, as many diverse data-sets as possible are needed: astrometry and parallaxes, photometry, polarimetry, spectroscopy — the more the better. Different physical and geometrical parameters, and their inter-dependencies, are revealed by different types of data-sets.

If we take all of the above into account, we may only conclude that follow-up observations are still badly needed.

\section{Conclusions and prospects for the future}

Overwhelming data quantities are upon us and changing traditional ways of modeling and analysis of eclipsing binaries is thus inevitable. There are many fine studies that bring us closer to this goal. One of the greatest properties of astronomy, when compared to other sciences, is a strong sense of collaboration, and absence of blind competition, between astronomers; our questions, therefore, on how to facilitate and how to propagate the idea of joint development of these new approaches, and how to handle huge data-sets that are pouring in, are aimed at every single individual interested in contributing its own piece to this fascinating puzzle. These are indeed scientifically challenging times and it would be too bad if we missed them.

\section{References}

Alcock, C., Allsman, R.A., Alves, D., Axelrod, T.S., Becker, A.C., Bennett, D.P., Cook, K.H., Freeman, K.C., Griest, K., Lacy, C.H.S., Lehner, M.J., Marshall, S.L., Minniti, D., 
Peterson, B.A., Pratt, M.R., Quinn, P.J., Rodgers, A.W., Stubbs, C.W., Sutherland, W., \& Welch, D.L. 1997, AJ, 114, 326

Andersen, J., Clausen, J.V., Nordstroem, B., \& Reipurth, B. 1983, A\&A, 121, 271

Baglin, A., Auvergne, M., Barge, P., Buey, J.-T., Catala, C., Michel, E., Weiss, W., \& COROT Team. 2002, in Stellar Structure and Habitable Planet Finding, ESA SP-485, ed. B. Battrick, F. Favata, I.W. Roxburgh, \& D. Galadi, 17-24

Brett, D.R., West, R.G., \& Wheatley, P.J. 2004, MNRAS, 353, 369

Bruntt, H., Southworth, J., Torres, G., Penny, A.J., Clausen, J.V., \& Buzasi, D.L. 2006, Aø A, 456,651

Cook, K.H., Alcock, C., Allsman, H.A., Axelrod, T.S., Freeman, K.C., Peterson, B.A., Quinn, P.J., Rodgers, A.W., Bennett, D.P., Reimann, J., Griest, K., Marshall, S.L., Pratt, M.R., Stubbs, C.W., Sutherland, W., \& Welch, D.L. 1995, in IAU Colloq. 155, Astrophysical Applications of Stellar Pulsation, ASP Conf. Ser. 83, ed. R. Stobie \& P. Whitelock, 221

Devinney, E., Guinan, E., Bradstreet, D., DeGeorge, M., Giammarco, J., Alcock, C., \& Engle, S. $2005, B A A S, 1212$

Devor, J. 2005, ApJ, 628, 411

Droege, T.F., Richmond, M.W., Sallman, M.P., \& Creager, R.P. 2006, ArXiv Astrophysics e-prints

Etzel, P.B. 1981, in Photometric and Spectroscopic Binary Systems, ed. E.B. Carling \& Z. Kopal, 111

Eyer, L. \& Blake, C. 2005, MNRAS, 358, 30

Friedemann, C., Guertler, J., \& Loewe, M. 1996, A\&AS, 117, 205

Graczyk, D. 2003, MNRAS, 342, 1334

Grison, P., Beaulieu, J.-P., Pritchard, J.D., Tobin, W., Ferlet, R., Vidal-Madjar, A., Guibert, J., Alard, C., Moreau, O., Tajahmady, F., Maurice, E., Prevot, L., Gry, C., Aubourg, E., Bareyre, P., Brehin, S., Gros, M., Lachieze-Rey, M., Laurent, B., Lesquoy, E., Magneville, C., Milsztajn, A., Moscoso, L., Queinnec, F., Renault, C., Rich, J., Spiro, M., Vigroux, L., Zylberajch, S., Ansari, R., Cavalier, F., \& Moniez, M.1995, A\& AS, 109, 447

Groenewegen, M.A.T. 2005, A\& A, 439, 559

Hacking, P., Lonsdale, C., Gautier, T., Herter, T., Shupe, D., Stacey, G., Fang, F., Xu, C., Graf, P., Werner, M., Soifer, B., Moseley, H., \& Houck, J. 1999, in Astrophysics with Infrared Surveys: A Prelude to SIRTF, ASP Conf. Ser. 177, ed. M.D. Bicay, R.M. Cutri, \& B.F. Madore, 409

Hadrava, P. 1990, Contributions of the Astronomical Observatory Skalnate Pleso, 20, 23

Hilditch, R.W., Harries, T.J., \& Howarth, I.D. 2004, New Astronomy Review, 48, 687

Hilditch, R.W., Howarth, I.D., \& Harries, T.J. 2005, MNRAS, 357, 304

Hill, G. 1979, Publications of the Dominion Astrophysical Observatory Victoria, 15, 297

Horák, T. 1966, Bulletin of the Astronomical Institutes of Czechoslovakia, 17, 27

Horák, T.B. $1970, A J, 75,1116$

Kaiser, N., Aussel, H., Burke, B.E., Boesgaard, H., Chambers, K., Chun, M.R., Heasley, J.N., Hodapp, K.-W., Hunt, B., Jedicke, R., Jewitt, D., Kudritzki, R., Luppino, G.A., Maberry, M., Magnier, E., Monet, D.G., Onaka, P.M., Pickles, A.J., Rhoads, P.H.H., Simon, T., Szalay, A., Szapudi, I., Tholen, D.J., Tonry, J.L., Waterson, M., \& Wick, J. 2002, in Survey and Other Telescope Technologies and Discoveries, Proceedings of the SPIE, Volume 4836, ed. J.A. Tyson \& S. Wolff, 154-164

Kholopov, P.N., Samus, N.N., Durlevich, O.V., Kazarovets, E.V., Kireeva, N.N., \& Tsvetkova, T.M. 1992, Bulletin d'Information du Centre de Donnees Stellaires, 40, 15

Koch, D.G., Borucki, W., Dunham, E., Geary, J., Gilliland, R., Jenkins, J., Latham, D., Bachtell, E., Berry, D., Deininger, W., Duren, R., Gautier, T.N., Gillis, L., Mayer, D., Miller, C.D., Shafer, D., Sobeck, C.K., Stewart, C., \& Weiss, M. 2004, in Optical, Infrared, and Millimeter Space Telescopes. Proceedings of the SPIE, Volume 5487, ed. J.C. Mather, 1491-1500

Lammers, U. 2005, in ESA SP-576: The Three-Dimensional Universe with Gaia, ed. C. Turon, K.S. O'Flaherty, \& M.A.C. Perryman, 445

Lastennet, E. \& Valls-Gabaud, D. 2002, A\& A, 396, 551

Linnell, A.P. \& Hubeny, I. 1994, ApJ, 434, 738

Maceroni, C., \& Rucinski, S.M. 1999, AJ, 118, 1819

Mazeh, T., Tamuz, O., \& North, P. 2006a, MNRAS, 367, 1531

-. 2006b, Ap\&SSS, 44 
Michalska, G. \& Pigulski, A. 2005, A\&A, 434, 89

Mochnacki, S.W. \& Doughty, N.A. 1972, MNRAS, 156, 51

Munari, U., Tomov, T., Zwitter, T., Milone, E.F., Kallrath, J., Marrese, P.M., Boschi, F., Prša, A., Tomasella, L., \& Moro, D. 2001, A\&A, 378, 477

Naficy, K., Riazi, N., \& Kiasatpour, A. 2005, AJ, 130, 1862

Nelson, B. \& Davis, W.D. 1972, ApJ, 174, 617

Neugebauer, G., Habing, H.J., van Duinen, R., Aumann, H.H., Baud, B., Beichman, C.A., Beintema, D.A., Boggess, N., Clegg, P.E., de Jong, T., Emerson, J.P., Gautier, T.N., Gillett, F.C., Harris, S., Hauser, M.G., Houck, J.R., Jennings, R.E., Low, F.J., Marsden, P.L., Miley, G., Olnon, F.M., Pottasch, S.R., Raimond, E., Rowan-Robinson, M., Soifer, B.T., Walker, R.G., Wesselius, P.R., \& Young, E. 1984, ApJL, 278, L1

Paczyński, B., Szczygieł, D.M., Pilecki, B., \& Pojmański, G. 2006, MNRAS, 368, 1311

Palanque-Delabrouille, N., Afonso, C., Albert, J.N., Andersen, J., Ansari, R., Aubourg, E., Bareyre, P., Bauer, F. et al. \& the EROS Collaboration. 1998, A\& A, 332, 1

Payne-Gaposchkin, C.H. 1971, The variable stars of the Large Magellanic Cloud, (Smithsonian Contributions to Astrophysics, Washington: Smithsonian Institution Press, - c1971)

Perryman, M.A.C., de Boer, K.S., Gilmore, G., Høg, E., Lattanzi, M.G., Lindegren, L., Luri, X., Mignard, F., Pace, O., \& de Zeeuw, P.T. 2001, A\&A, 369, 339

Perryman, M.A.C. \& ESA. 1997, The Hipparcos and Tycho catalogues. Astrometric and photometric star catalogues derived from the ESA Hipparcos Space Astrometry Mission, Publisher: Noordwijk, Netherlands: ESA Publications Division, Series: ESA SP Series vol no: 1200, ISBN: 9290923997 (set)

Pojmanski, G. 1997, Acta Astronomica, 47, 467

-. 2002, Acta Astronomica, 52, 397

Prša, A. 2003, in GAIA Spectroscopy: Science and Technology, ASP Conf. Ser. 298, ed. U. Munari, 457

Prša, A. \& Zwitter, T. 2005a, ApJ, 628, 426

-. 2005b, Ap\&SSS, 296, 315

Prša, A., \& Zwitter, T. 2005c, in The Three-Dimensional Universe with Gaia, ESA SP-576, ed. C. Turon, K.S. O'Flaherty, \& M.A.C. Perryman, 611

-. 2006, ArXiv Astrophysics e-prints

Ribas, I., Jordi, C., Vilardell, F., Giménez, Á., \& Guinan, E.F. 2004, New Astronomy Review, 48,755

Rucinski, S.M. 1973, Acta Astronomica, 23, 79

Rucinski, S.M. 1997a, $A J, 113,1112$

-. 1997b, AJ, 113, 407

-. 1998, AJ, 115, 1135

Rucinski, S.M. \& Maceroni, C. 2001, AJ, 121, 254

Sarro, L.M., Sánchez-Fernández, C., \& Giménez, Á. 2006, A\&A, 446, 395

Szymanski, M., Udalski, A., Kubiak, M., Kaluzny, J., Mateo, M., \& Krzeminski, W. 1996, Acta Astronomica, 46, 1

Tamuz, O., Mazeh, T., \& North, P. 2006, MNRAS, 367, 1521

Tamuz, O., Mazeh, T., \& Zucker, S. 2005, MNRAS, 356, 1466

Tody, D. 1986, in Proceedings of Instrumentation in astronomy VI; Part 2 (A87-36376 15-35). Bellingham, WA, Society of Photo-Optical Instrumentation Engineers, ed. D.L. Crawford, 733

Udalski, A., Kubiak, M., \& Szymanski, M. 1997, Acta Astronomica, 47, 319

Wilson, R.E. 2004, New Astronomy Review, 48, 695

Wilson, R.E. \& Devinney, E.J. 1971, ApJ, 166, 605

Wood, D.B. 1971, AJ, 76, 701

Wyithe, J.S.B. \& Wilson, R.E. 2001, ApJ, 559, 260

—. 2002, ApJ, 571, 293

Wyrzykowski, L., Udalski, A., Kubiak, M., Szymanski, M., Zebrun, K., Soszynski, I., Wozniak, P.R., Pietrzynski, G., \& Szewczyk, O. 2003, Acta Astronomica, 53, 1

Wyrzykowski, L., Udalski, A., Kubiak, M., Szymanski, M.K., Zebrun, K., Soszynski, I., Wozniak, P.R., Pietrzynski, G., \& Szewczyk, O. 2004, Acta Astronomica, 54, 1 